\title{
The interactions of cetyltrimethylammonium with mitochondria: an uncoupler or a detergent?
}

\author{
Marcantonio Bragadin ${ }^{\mathrm{a}, *}$, Ivo Moret ${ }^{\mathrm{b}}$, Rossano Piazza ${ }^{\mathrm{a}}$, \\ Mario Grasso ${ }^{\text {a }}$, Sabrina Manente ${ }^{\mathrm{a}}$ \\ a Dipartimento di Scienze Ambientali, Università di Venezia, DD 2137, 30123 Venice, Italy \\ ${ }^{\mathrm{b}}$ CNR Centro di Studio sulla Chimica e le Tecnologie per l'ambiente, DD 2137, 30123 Venice, Italy
}

Received 6 November 2000; accepted 5 March 2001

\begin{abstract}
The interactions of cetyltrimethylammonium (CTA) with mitochondria have been investigated. We confirm, as already observed in a previous paper, that this compound behaves as proton carrier (or uncoupler) of the oxidative phosphorylation, but evidences suggest that this compound enhances the membrane permeability to many other compounds such as sucrose. We conclude therefore that CTA as a detergent enhances membrane permeability to all ions including protons. Some evidences are also given that the inhibitory effect of CTA on the mitochondrial respiratory chain is a consequence of the swelling induced. (c) 2002 Elsevier Science Ltd. All rights reserved.
\end{abstract}

Keywords: Mitochondria; Toxicity; Detergents

\section{Introduction}

Detergents are a class of pollutants of greatest environmental concern. The anionic and neutral ones are the most commonly employed and their effects on living organisms have been extensively investigated (Linfield, 1976; Potokar, 1980; Fell, 1991). The cationic detergents are increasingly utilized because of their softening properties.

The toxic effects of detergent compounds have been studied not only in whole animals, but also by experimenting in vitro conditions using cells and subcellular structures in order to establish the molecular mechanism

\footnotetext{
*Corresponding author. Tel.: +39-041-2578511; fax: +39041-2578584.

E-mail address: bragadin@unive.it (M. Bragadin).
}

which is responsible for the effects in the whole animals. Mitochondria are the preferential target of many toxic compounds (Higgins and Rogers, 1974; Bragadin and Dell'Antone, 1996; Bragadin and Marton, 1997a; Bragadin and Viola, 1997b; Bragadin et al., 1998, 1999) since a damage to mitochondria which synthesize ATP gives rise to a corresponding cell damage. In particular, the interactions of cationic detergents with mitochondria have been studied. In their report, Higgins and Rogers (1974) conclude that CTA is an inhibitor of ATP synthesis acting as an uncoupler of the oxidative phosphorylation. In a subsequent paper (Bragadin and Dell'Antone, 1996), a different interaction mechanism has been proposed: CTA is accumulated inside the mitochondria being attracted by the negative-inside potential. Once accumulated, CTA induces a membrane leak with loss of all ions. In the present paper, we give further evidences in favour of this mechanism, which is different from the uncoupling one. By this mechanism, also the already observed mitochondrial respiratory chain inhibition is easily explained. 


\section{Materials and methods}

Mitochondria were prepared from livers of fasted male Albino Winstar rats weighing about 300 g. The livers were isolated and placed in ice-cold $0.25 \mathrm{M}$ sucrose, $10 \mathrm{mM}$ Tris-buffer at $\mathrm{pH}$ 7.4, and $0.1 \mathrm{mM}$ EGTA. They were rinsed three times with ice-cold medium, cut into small cubes with scissors, and homogenized in a potter homogenizer fitted with a rotating teflon pestle in an ice-water bath ( 2 strokes with $40 \mathrm{ml}$ medium per liver sample). The homogenate was diluted to approximately $200 \mathrm{ml}$ per sample, and unbroken cells and nuclei were sedimented by centrifugation at $650 \times g$ in a Sorvall $\mathrm{RC} 2 \mathrm{~B}$ refrigerated centrifuge at $2^{\circ} \mathrm{C}(\mathrm{GSA}$ rotor at 2000 $\mathrm{rpm}$ for $10 \mathrm{~min}$ ). The supernatant was carefully decanted and centrifuged at $7800 \mathrm{rpm}$ for $10 \mathrm{~min}$. It was then discarded and the mitochondrial pellet was carefully resuspended in an ice-cold medium and spun as above. The resulting pellet was resuspended in $0.25 \mathrm{M}$ sucrose and $10 \mathrm{mM}$ Tris at $\mathrm{pH} 7.4$ and stored on ice at approximately $150 \mathrm{mg}$ protein/ml. Mitochondrial protein content was determined by the Lowry reaction (Lowry et al., 1951).

Swelling experiments were performed by measuring the absorbance decrease at $540 \mathrm{~nm}$ by a Jenway 6400 Spectrophotometer. After addition of the mitochondria to the medium, the instrument was reset to zero absorbance. Therefore the absorbance lowering appeared as a negative value. When monitoring endogenous $\mathrm{K}^{+}$and $\mathrm{Ca}^{++}$released from non-energized mitochondria in the medium (Fig. 2), the mitochondria (final concentration, $0.5 \mathrm{mg} / \mathrm{ml}$ ) were added to the incubating medium. In the experiments performed in the absence of cyclosporine (experiments A), the medium composition was: $0.25 \mathrm{M}$ sucrose, $10 \mathrm{mM}$ Tris $\mathrm{pH}$ 7.4. In the experiments performed in the presence of cyclosporine (experiments B), the medium composition was: $0.25 \mathrm{M}$ sucrose, $10 \mathrm{mM}$ Tris $\mathrm{pH} 7.4,2 \gamma$ cyclosporine A. The medium containing mitochondria was subdivided in five samples (experiment A), $4 \mathrm{ml}$ each. In the first, second, third, and fourth sample, CTA (sample b), sodiumdodecylsulphate (SDS) (sample c), triton (sample d) and phenylarsine (PhA) (sample e) was added, respectively. Last, a reference sample with no chemical compound was prepared (sample a). Then, all five samples were repeated in the presence of cyclosporine also (experiments B). After 10 min incubation, each sample was centrifuged at 15000 rpm (rotor SS34, Sorvall RC2B centrifuge) for $10 \mathrm{~min}$. The supernatant concentration of $\mathrm{K}^{+}$and $\mathrm{Ca}^{++}$was measured by a flame spectroscopy.

Respiratory rate experiments were performed by a Clark selective oxygen electrode thermostated at $20^{\circ} \mathrm{C}$. Cetyltrimethylammoniumbromide (hexadecyltrimethylammoniumbromide) (CTA), sodiumdodecylsulphate (SDS), triton (t-octylphenoxypolyethoxyethanol), phenylarsine $(\mathrm{PhA})$ and 2,4 dinitrophenol were obtained from
Sigma, Milan. All chemicals were of the highest purity, commercially available.

\section{Results and discussion}

In mitochondria, substrates arising from the Krebs cycle are oxidized by molecular oxygen through the respiratory chain. In the respiratory chain the electron flow is coupled to a proton pump and protons are extruded. According to the Mitchell's chemiosmotic hypothesis (Mitchell, 1979), the mitochondrial membrane is not permeable to protons. Therefore, protons extrusion gives rise to a $\Delta \mathrm{pH}$ (alkaline-inside) and to a $\Delta \Psi$ (negative-inside). The protonmotive force (p.m.f. $=$ $\Delta \mathrm{pH}+\Delta \Psi)$ is the high energy intermediate which transforms ADP in ATP. The chemiosmotic hypothesis can explain also the mechanism of uncouplers: the uncouplers are chemical compounds at low doses inhibiting ATP synthesis. They are weak acids which enter the mitochondria as electroneutral compounds and tend to accumulate in the matrix by the alkaline-inside $\mathrm{pH}$. Once inside, the uncouplers are extruded as anions. The whole balance of the cyclic mechanism is the transport of a proton through the membrane at any cycle. In this condition the membrane is no longer able to store energy as $\Delta \mathrm{pH}$ and $\Delta \Psi$ with consequent failure in ATP synthesis.

This mechanism cannot be proposed to explain some of the interactions of CTA with the mitochondria and in particular its uncoupling effect, since CTA is not a weak acid. We have already evidenced this situation in a previous paper (Bragadin and Dell'Antone, 1996), demonstrating, by a selective electrode that CTA is captured by the negative potential present inside mitochondria and then released. In fact, the uptake and initial accumulation gives rise to a detergent effect causing membrane leak and $\mathrm{K}^{+}$and $\mathrm{Ca}^{++}$loss. In this paper, we give further evidences about this mechanism, thus supporting the idea that the enhancement of proton conductivity in mitochondria is only a particular aspect of a more general behaviour, i.e., a detergent effect. This involves a permeability enhancement to all chemical compounds, protons included. In particular, in the present paper, we give evidences by a different procedure, that CTA induces sucrose transport in the mitochondrial membrane. It is to remark that sucrose is not permeant in mitochondria and that sucrose is a high molecular weight molecule.

For this purpose, non-respiring and non-energized mitochondria were resuspended in an isoosmotic sucrose medium. If CTA is added, swelling occurs (Fig. 1(a)). The swelling was monitored as absorbance decrease at $540 \mathrm{~nm}$ and was due to the entry of solute. While the sucrose enters, many ions and macromolecules in the matrix, because of a colloid process, cannot exit. In 


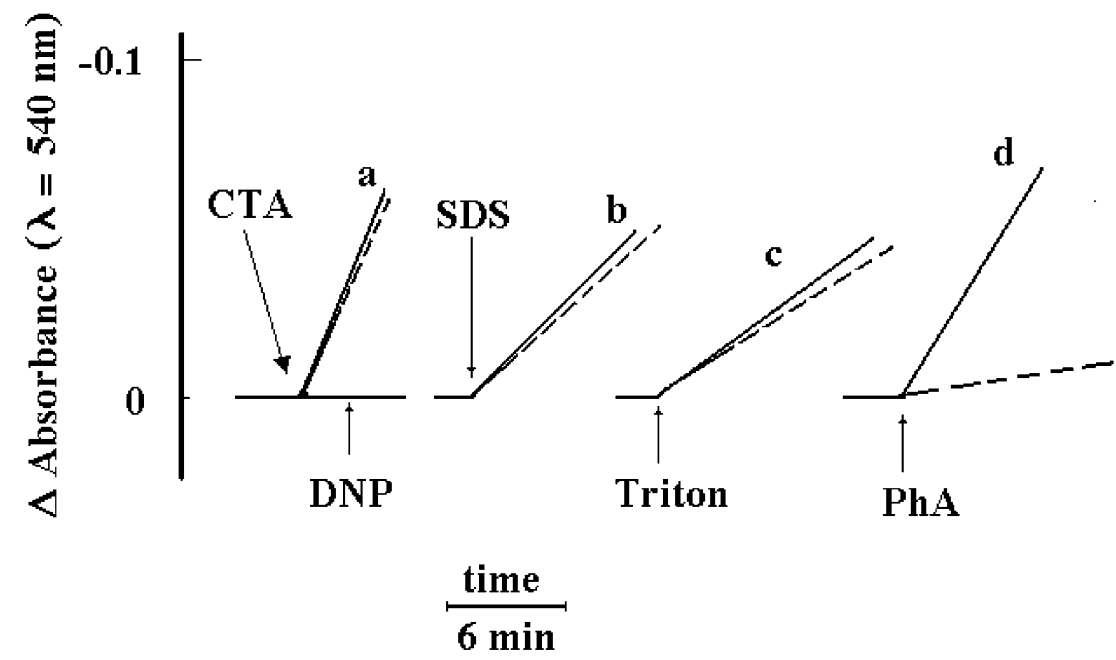

Fig. 1. Mitochondrial swelling induced by SDS, CTA, triton and PhA. To the operating medium (medium composition: $0.25 \mathrm{M}$ sucrose, $10 \mathrm{mM}$ Tris $\mathrm{pH}$ 7.4), mitochondria were added and the final concentration of mitochondria was $0.5 \mathrm{mg} / \mathrm{ml}$. The instrument was then adjusted at absorbance $=0$ at $540 \mathrm{~nm}$. In (a) CTA $(20 \mu \mathrm{m})$, in (b) SDS $(4 \mu \mathrm{M})$, in (c) triton $(1 \mathrm{ppm})$ and in (d) phenylarsine (PhA) $20 \mu \mathrm{M}$ were added. The dotted line shows the same experiment performed in the presence of $2 \gamma$ cyclosporine A, the inhibitor of the opening of the permeability transition pore, MPT. (a) shows that $100 \mu \mathrm{M} 2,4$ dinitrophenol (DNP) does not induce any swelling, either in the presence or in the absence of cyclosporine, thus supporting the hypothesis that the effect of CTA is a detergent and not an uncoupling effect.

order to ensure the osmotic balance, the sucrose entry is accompanied by water entry and the mitochondria swell (Azzone and Azzi, 1965).

In general, the swelling may be due to many causes.

(a) The swelling is due to the opening of a permeability transition pore (MPT). This phenomenon corresponds to the opening of a pore which enables the free diffusion of solutes with a molecular mass up to $1500 \mathrm{Da}$ (Bernardi et al., 1994; Bernardi, 1999; Zoratti and Szabo, 1995). Many chemical compounds induce this kind of permeability and consequent swelling. Fig. 1(d) shows the swelling induced by phenylarsine ( $\mathrm{PhA}$ ), a potent inducer of the opening of the MPT pore (Zoratti and Szabo, 1995). The swelling is inhibited by cyclosporine $\mathrm{A}$, as in all cases in which the swelling is due to the MPT pore.

(b) The swelling is induced by detergent compounds. All detergent compounds (anionic, cationic and neutral) possess an hydrophobic chain which interacts with the hydrophobic chain of the phospholipidic membrane. The interaction causes permeability loss, sucrose entry and consequent swelling. The swelling is a specific and it is not inhibited by cyclosporine $\mathrm{A}$, as shown in the examples of Figs. 1(b) and (c).

(c) Swelling is due to the presence in the mitochondrial membrane of antiporters (Bernardi, 1999). The native $\mathrm{Na}^{+} / \mathrm{H}^{+}$antiporter, for example, induces swelling in the presence of sodium acetate (Bernardi, 1999). Analogously, the $\mathrm{Ca}^{++} / \mathrm{H}^{+}$antiporter, A23187, induces swelling providing the presence of calcium acetate (Bernardi,
1999). In all these kinds of experiments, the swelling is induced providing the presence not only of an antiporter, but also of the corresponding ions, such as $\mathrm{Na}^{+}$ in the example above cited. This kind of swelling is not inhibited by cyclosporine A.

The swelling observed after addition of CTA as in Fig. 1(a) cannot be ascribed to the mechanism c since the antiporter which should regulate the CTA uptake does not exist. Furthermore, no ions are present in the medium which could activate any antiporter system (the medium contain only sucrose and buffer).

A comparison of the behaviour of CTA with that of SDS (Fig. 1(b)), triton (Fig. 1(c)) and PhA (Fig. 1(d)) suggests that the behaviour of CTA is similar to that of the detergent compounds SDS and triton since, differently from PhA (Fig. 1(d)), the swelling is not inhibited by cyclosporine $\mathrm{A}$.

In this regard, a further evidence arises from experiments in which the $\mathrm{K}^{+}$and $\mathrm{Ca}^{++}$concentrations in the supernatant of non-respiring mitochondria after addition of SDS, triton, PhA and CTA, were measured. Results indicate (Fig. 2) that CTA behaves like SDS and triton since CTA induces a $\mathrm{K}^{+}$and $\mathrm{Ca}^{++}$release which is not cyclosporine sensitive (histogram A), while in the case of PhA only a limited $\mathrm{K}^{+}$and $\mathrm{Ca}^{++}$release occurs if cyclosporine $\mathrm{A}$ is present (histogram $\mathrm{B}$ ).

We conclude that CTA is a detergent which enhances the membrane permeability to all solutes, protons included. In this regard, following our statement, it is to remark that the already observed proton permeability 

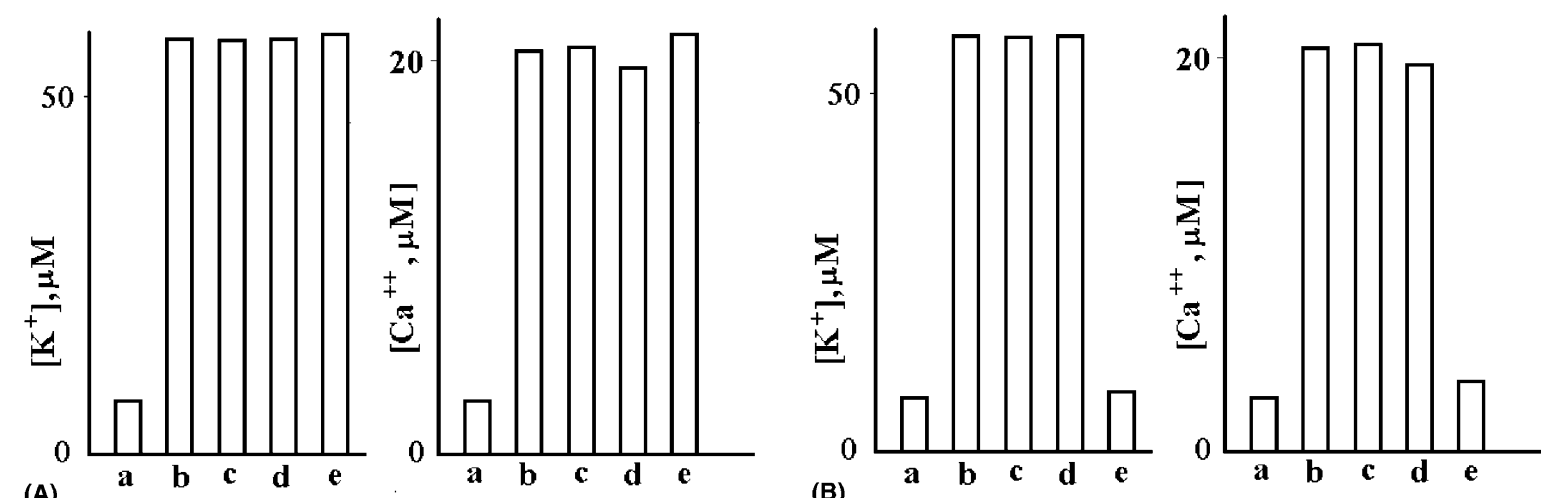

Fig. 2. Potassium and calcium in the supernatant of non-respiring mitochondria. The histograms show the concentration of $\mathrm{K}^{+}$and $\mathrm{Ca}^{++}$in the supernatant of non-respiring mitochondria in the absence (A) and in the presence (B) of cyclosporine A. Medium composition and mitochondrial concentration: in experiment $\mathrm{A}, 0.25 \mathrm{M}$ sucrose, $10 \mathrm{mM}$ Tris $\mathrm{pH}$ 7.4. in experiments $\mathrm{B}, 0.25 \mathrm{M}$ sucrose, $10 \mathrm{mM}$ Tris $\mathrm{pH}$ 7.4, $2 \gamma$ cyclosporine. In all experiments, the mitochondrial concentration was $0.5 \mathrm{mg} / \mathrm{ml}$. In (a) no additions of chemical compounds (reference sample). In (b) $20 \mu \mathrm{M} \mathrm{CTA}$, in (c) $4 \mu \mathrm{M}$ SDS, in (d) 1 ppm triton, in (e) $20 \mu \mathrm{M}$ PhA were added before incubation and centrifugation.

enhancement induced by CTA (Higgins and Rogers, 1974), is only a particular case of a more general membrane permeability enhancement to all solutes. Furthermore, CTA cannot be an uncoupler not only since it is not a weak acid, but an uncoupler (DNP), as shown in Fig. 1(a), does not induce any swelling.

According to the above-mentioned behaviour which causes swelling, the already observed respiratory chain inhibition (Higgins and Rogers, 1974) should not be due to a direct interaction with the mitochondrial respiratory chain, but it is an indirect consequence of the swelling which causes cytochrome $c$ release (Jacobs and Sanadi, 1960). This behaviour was verified by addition of cytochrome $c$ to mitochondria in the presence of CTA: CTA induces respiratory chain inhibition, but cytochrome $c$

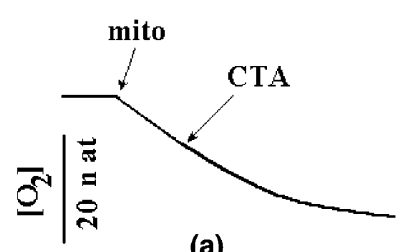

(a)

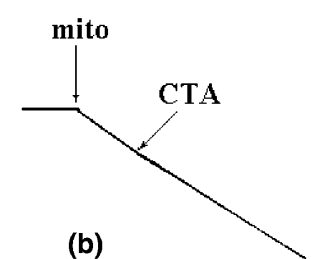

(b) restores the respiratory rate at values similar to that measured in the absence of CTA (Fig. 3).

\section{Conclusion}

In conclusion, our experiment about the entry of a big molecule such as sucrose gives new evidences in favour of a detergent effect, thus supporting the conclusion that the uncoupling effect (= proton permeability enhancement) is only a particular aspect of a more general effect induced by CTA. This compound, being a detergent, enhances the permeability to all solutes. In this regard, it has been demonstrated that CTA enters the mitochondria by a potential driven mechanism (Bragadin and Dell'Antone, 1996), but this mechanism does not exclude an interaction between the hydrophobic chains even in not energized conditions (see experiments of Fig. 2).

By respiratory rate experiments, it is possible to give evidence that the respiratory chain inhibition is a consequence of the swelling of mitochondria and it is not due to an interaction with a particular site in the respiratory chain.

\section{References}

Azzone, G.F, Azzi, A., 1965. Volume changes in liver mitochondria. Proc. Natl. Acad. Sci. USA 53, 1084-1089.

Bernardi, P., Brockmeier, K., Pfeiffer, D.R., 1994. Recent progress on regulation of the mitochondrial permeability transition pore, a cyclosporine-sensitive pore in the inner mitochondrial membrane. J. Bioenerg. Biomembr. 26, 509517.

Fig. 3. CTA induces respiratory chain inhibition and cytocomposition: $0.25 \mathrm{M}$ sucrose, $10 \mathrm{mM}$ Tris $\mathrm{pH} 7.4,2 \mathrm{mM}$ succinate. To the medium mitochondria were added and the final mitochondrial concentration was $0.5 \mathrm{mg} / \mathrm{ml}$. Arrows indicate the addition of $20 \mu \mathrm{M}$ CTA. In (b) the conditions same as in (a), but in the medium was present $0.2 \mathrm{mM}$ cytochrome $c$. 
Bernardi, P., 1999. Mitochondrial transport of cations: channels, exchangers and permeability transition. Physiol. Rev. 79, 1127-1155.

Bragadin, M., Dell'Antone, P., 1996. Mitochondrial bioenergetics as affected by cationic detergents. Arch. Environ. Contam. Toxicol. 30, 280-284.

Bragadin, M., Marton, D., 1997a. A proposal for a mechanism of interaction of trialkyltin (tat) compounds with mitochondria. J. Inorg. Biochem. 68, 75-78.

Bragadin, M., Viola, E.R., 1997b. $\mathrm{Ni}^{++}$as a competitive inhibitor of calcium transport in mitochondria. J. Inorg. Biochem 6, 227-229.

Bragadin, M., Marton, D., Murgia, M., Rizzoli, V., Scutari, G., Deana, R., 1998. J. Inorg. Biochem. 69, 259-262.

Bragadin, M., Perin, G., Iero, A., Manente, S., Rizzoli, V., Scutari, G., 1999. An in vitro study on the toxic effects of nonylphenols (NP) in mitochondria. Chemosphere 38, 1997-2001.

Fell, B., 1991. Tenside: actueller Stand-Absehbare entwicklungen. Tenside Surf. Det. 28, 385-395.

Potokar, M.S., 1980. Anionic Surfactants, Biochemistry, Toxicology, dermatology. In: Gloxhuber, C. (Ed.), Surfactant Science Series, vol. 10. Marcel Dekker, New York, p. 87.
Higgins, E.S., Rogers, K.S., 1974. Effects of cetyltrimethylammonium and dodecylnicotinamide halides on functional states of mitochondria. Proc. Soc. Exp. Biol. Med. 146, 8591.

Jacobs, E.E., Sanadi, D.R., 1960. The reversible removal of cytochrome $c$ from mitochondria. J. Biol. Chem. 235, 531534.

Linfield, W.M., 1976. Anionic Surfactants. Marcel Dekker, New York.

Lowry, O.H., Rosenbrough, N.Y., Farr, A.L., Randall, R.J., 1951. Protein measurement with folin phenol reagent. J. Biol. Chem. 193, 265-275.

Mitchell, P., 1979. Keilin' respiratory chain concept and its chemiosmotic consequences. Science 286, 1148-1159.

Zoratti, M., Szabo, S., 1995. The mitochondrial permeability transition. Biochim. Biophys. Acta 1241, 139-176.

Marcantonio Bragadin was born in Venice on 8/1/1947. In 1970, he graduated in physical chemistry from the University of Padova. Since 1983, he is an associate professor in Macromolecular Biochemistry at the University of Venice (Department of Environmental Sciences). Since 1990 he has been dealing with problems correlated with the toxicology in aqueous environment and with the action mechanisms of toxic compounds in whole cells, in mitochondria and acid vescicles. 